\section{Sabine Lorch}

\section{Einleitung}

Bei vielen pflanzlichen Arzneimitteln stellt sich - nicht zuletzt vonseiten der Zulassungsbehörden - immer wieder die Frage nach der Wirksamkeit und Sicherheit. Gerade im Hinblick auf Risikogruppen wie Schwangere, Kinder und Patienten mit schweren Begleiterkrankungen sind meist nur wenige Anwendungsdaten vorhanden. Dies erschwert ihre $\mathrm{Zu}$ lassung für diese Patientengruppen und damit deren Behandlung mit pflanzlichen Arzneimitteln, obwohl sich diese meist schon länger in der täglichen Praxis bewährt haben. Nicht nur in solchen Situationen, sondern auch bei der Verschreibung für nicht zugelassene Indikationen befindet sich der Arzt demnach im «Off-Label-Use».

Wegen des grossen finanziellen Aufwands kann jedoch nur zu manchen Präparaten eine klinische Studie durchgeführt werden. Die Kooperation Phytopharmaka in Deutschland (www.koop-phyto.org) hat deswegen durch einen gepoolten Ansatz eine einfache und kostengünstigere Lösung gesucht und das Projekt «Erfahrungsdatenbank Phytopharmaka» bzw. PhytoVis ins Leben gerufen, an dem sich auch die Schweizerische Medizinische Gesellschaft für Phytotherapie (SMGP) beteiligt. Zusammen mit Prof. Dr. Ralph Mösges vom Institut für medizinische Statistik, Informatik und Epidemiologie (IMSIE) der Universität Köln wurde ein Fragebogen entwickelt, mit dem in Arztpraxen und Apotheken online die

\title{
Pflanzliche Arzneimittel: Mehr Daten zu Wirksamkeit und Sicherheit dank einfacher Online- Dokumentation mit «PhytoVis»
}

Anwendung von pflanzlichen Arzneimitteln fallbezogen dokumentiert werden kann.

\section{Das Kernstück von PhytoVis: \\ Der Online-Fragebogen}

Der Online-Fragebogen eignet sich zur Untersuchung vieler Fragestellungen, insbesondere bei Risikogruppen wie Kindern, Schwangeren/Stillenden und älteren Personen. Ein weiterer Vorteil ist, dass er auch auf Wirkstoffebene (d.h. produktübergreifend) zum Einsatz kommen kann. Die Eingabezentren können zudem periodisch auf bestimmte Schwerpunktthemen sensibilisiert werden. Auf der Basis der wachsenden Datenmenge wird nach und nach das Beantworten komplexerer Fragestellungen möglich werden. Die strukturierte Erfassung durch medizinische Fachpersonen sichert eine zuverlässige Qualität der Daten.

In einer Evaluierungsphase wurden im Januar/Februar 2012 mit mehreren Eingabezentren (Arztpraxen) die Bedienerfreundlichkeit der Online-Version und die Verständlichkeit der Fragen erfolgreich getestet. Nach dem zustimmenden Votum der Ethikkommission Köln am 28. März 2012 konnte PhytoVis am 5. Juli 2012 in die Pilotphase starten.

Jedes Eingabezentrum erhält nach der Anmeldung einen persönlichen, direkten Link zum Online-Fragebogen. Erfasst werden retrospektiv phytotherapeutische Behandlungen, die nicht länger als 8 Wochen zurück- liegen bzw. deren letzte Anwendung nicht länger her ist. Wesentlich für die verlässliche Qualität der Daten ist, dass der Patient seine Meinung zu Wirkung, unerwünschten Wirkungen und Zufriedenheit mit dem pflanzlichen Arzneimittel angibt, der Arzt oder Apotheker mit ihm aber die einzelnen Fragen durchgeht und die Ergebnisse letztendlich aus fachlicher Sicht beurteilt und online eingibt.

\section{Patient}

Erfasst werden Begleiterkrankungen und Begleitmedikation, Altersgruppe und Geschlecht; bei Frauen wird nach der Anwendung während Schwangerschaft oder Stillzeit gefragt. Anzumerken ist, dass in der Pilotphase noch nicht aktiv auf spezielle Patientengruppen fokussiert wird.

\section{Indikation}

Aus einer Liste wird die Indikationsgruppe für die Anwendung des pflanzlichen Arzneimittels ausgewählt. Für andere Indikationen steht ein Freitextfeld zur Verfügung, sodass auch seltene Indikationen somit dokumentiert werden können.

\section{Arzneimittel}

Die aktuellen Arzneimittellisten, die in den Fragebogen integriert sind (pflanzliche Arzneimittel gemäss Bundesvereinigung Deutscher Apothekerverbände (ABDA) bzw. Swissmedic), erleichtern die genaue Erfassung des Präparats. Das Freitextfeld dazu eignet sich für Magistralrezepturen, Hausspezialitäten, pflanzliche

\section{KARGER}

Fax +4976145207 14
(๑) 2013 S. Karger GmbH, Freiburg

Accessible online at: www.karger.com/szg
Sabine Lorch, FPH Offizinpharmazie

Schweizerische Medizinische Gesellschaft für Phytotherapie (SMGP)

Seestrasse 366, 8038 Zürich, Schweiz

phytonetz@yahoo.com 
Drogen und Zubereitungen (z.B. Tinkturen), die offen abgefüllt werden, und ebenso für Präparate, die von der Behörde nicht als pflanzliches Arzneimittel eingestuft werden, aber aus wissenschaftlicher Sicht als solche angesehen werden müssen. Dazu zählen auch bestimmte Nahrungsergänzungen - ein wichtiger Punkt, da mehr und mehr Hersteller mit ihren pflanzlichen Produkten in diesen Bereich ausweichen, weil das aufwendige Registrierungsverfahren für Heilmittel $\mathrm{zu}$ teuer wird.

\section{Wirksamkeit und Verträglichkeit}

Neben der Frage nach unerwünschten Wirkungen, ihrer Art und ihrer Dauer, und ob sie eine Arztkonsultation erforderlich gemacht haben, werden in einem Raster gleichzeitig die Wirksamkeit und die Verträglichkeit beurteilt, wobei für jedes Kriterium 4 Stufen zur Verfügung stehen. So ergeben sich 16 Möglichkeiten zur differenzierten Beurteilung.

\section{Empfehlung und Bezugsquelle \\ Wenn pflanzliche Arzneimittel auch häufig von medizinischen Fachper- sonen empfohlen werden, sind weite- re Quellen möglicherweise wichtiger, als mancher Arzt oder Apotheker denkt. Bei der Bezugsquelle stehen}

Apotheke und Drogerie sicher an vorderer Stelle. Interessant dürfte jedoch sein, welche Rolle Supermarkt, Internet und andere spielen.

\section{Kommentare von Therapeut und/oder Patient}

Das Freitextfeld «Kommentar» am Ende des Fragebogens ergänzt die standardisierten Fragen. Neben der persönlichen Meinung können z.B. weitere Details zum dokumentierten Fall vermerkt werden. Ebenso können Einzelheiten zu Magistralrezepturen oder zu speziellen Produkten hier angegeben werden.

\section{Wie geht es weiter?}

Die Pilotphase ist gut angelaufen und wird seit März 2013 evaluiert, während die Datenerfassung natürlich weiterläuft. Dank der sorgfältigen Vorbereitung und Betreuung durch die Kooperation Phytopharmaka und dem von Prof. Dr. Mösges an der Universität Köln eigens dafür entwickelten, durchdachten OnlineErfassungstool lassen sich phytotherapeutische Anwendungsbeobachtungen nicht nur rasch und unkompliziert erfassen, sondern die standardisierten Fragen und Indikationslisten ermöglichen auch eine

\section{PhytoVis braucht Sie!}

Eine erfreuliche Anzahl von Arztpraxen und Apotheken beteiligt sich inzwischen an dem Projekt, und es ist ihr Engagement, das ganz wesentlich zum Aufbau und zur Weiterentwicklung der dringend nötigen «Erfahrungsdatenbank Phytopharmaka» beiträgt!

Um aussagekräftige Datenmengen zusammenzutragen, sind jedoch noch viel mehr aktive Eingabezentren erforderlich. ÄrztInnen und ApothekerInnen, die bereit sind, eigene Erfahrungen mit pflanzlichen Arzneimitteln auf diese Weise zu dokumentieren, finden das Anmeldeformular unter www.phytovis.de oder auf der Internetseite der SMGP unter www.smgp.ch.

differenzierte Auswertung $\mathrm{zu}$ bestimmten Fragestellungen.

Geplant ist, in Zukunft den Schwerpunkt der Datensammlung vermehrt auf gezielte Fragestellungen und spezielle Patientengruppen (Kinder, Schwangere/Stillende und ältere Personen) zu legen. Parallel dazu soll die allgemeine Erfassung von phytotherapeutischen Anwendungen aus der Arztpraxis oder Apotheke fortgesetzt werden. Vorbehalten bleibt der Entscheid des wissenschaftlichen Beirats der Kooperation Phytopharmaka nach der Auswertung der Pilotphase.

\section{Fazit: PhytoVis - Fakten helfen heilen}

Ohne eine detaillierte Dokumentation zur Wirksamkeit und Sicherheit haben pflanzliche Arzneimittel einen schweren Stand. Dies zeigt sich etwa bei der aktuellen Diskussion um die Anwendung von Suppositorien mit ätherischen Ölen im Säuglings- und Kleinkindesalter (siehe auch [1]). Damit diese bewährte Therapieoption bestehen bleibt, sind Anwendungsdaten zur Sicherheit von ätherischen Ölen bei Kindern unter 30 Monaten notwendig - was bisher zu wenig dokumentiert wurde.

Die Erfahrungsdatenbank PhytoVis möchte hier Abhilfe schaffen. Je mehr Arztpraxen und Apotheken sich am Projekt beteiligen (siehe Kasten), umso rascher und sicherer wächst die Menge der Informationen - damit pflanzlichen Arzneimitteln aufgrund der Fakten der Stellenwert in der Therapie gesichert werden kann, der ihnen zukommt.

\section{Literatur}

1 Falch B, Kramer B: Verwendung von Zäpfchen mit ätherischen Ölen im Säuglings- und Kleinkindesalter. Forsch Komplementmed 2012;19:330-332. 